\title{
Role of precision imaging in esophageal cancer
}

\author{
Sherif B. Elsherif ${ }^{1,2}$, Sonia Andreou ${ }^{1}$, Mayur Virarkar $^{2}$, Erik Soule ${ }^{1}$, Dheeraj Reddy Gopireddy ${ }^{1}$, \\ Priya R. Bhosale ${ }^{2}$, Chandana Lall ${ }^{1}$
}

${ }^{1}$ Department of Radiology, University of Florida College of Medicine, Jacksonville, FL, USA; ${ }^{2}$ Department of Diagnostic Radiology, The University of Texas MD Anderson Cancer Center, Houston, TX, USA

Contributions: (I) Conception and design: All authors; (II) Administrative support: None; (III) Provision of study materials or patients: None; (IV) Collection and assembly of data: All authors; (V) Data analysis and interpretation: All authors; (VI) Manuscript writing: All authors; (VII) Final approval of manuscript: All authors.

Correspondence to: Sherif B. Elsherif, MD. Department of Radiology, University of Florida College of Medicine, Jacksonville, FL, USA.

Email: sherif.b.elsherif@gmail.com.

\begin{abstract}
Esophageal cancer is a major cause of morbidity and mortality worldwide. Recent advancements in the management of esophageal cancer have allowed for earlier detection, improved ability to monitor progression, and superior treatment options. These innovations allow treatment teams to formulate more customized management plans and have led to an increase in patient survival rates. For example, in order for the most effective management plan to be constructed, accurate staging must be performed to determine tumor resectability. This article reviews the multimodality imaging approach involved in making a diagnosis, staging, evaluating treatment response and detecting recurrence in esophageal cancer.
\end{abstract}

Keywords: Esophageal cancer; esophageal adenocarcinoma (EAC); esophageal squamous cell carcinoma (ESCC); MRI; endoscopic ultrasonography

Submitted Jul 15, 2019. Accepted for publication Jul 29, 2019.

doi: $10.21037 /$ jtd.2019.08.15

View this article at: http://dx.doi.org/10.21037/jtd.2019.08.15

\section{Introduction}

Esophageal cancer is the eighth most common cancer and the sixth most common cause of cancer-related deaths worldwide (1) with approximately 17,000 new cases diagnosed every year in the United States (2-4). The two major histologic subgroups diagnosed in esophageal cancer are squamous cell carcinoma and adenocarcinoma. Esophageal squamous cell carcinoma (ESCC) typically arises in the middle and lower thirds of the esophagus, with only $10-15 \%$ occurring in the upper one-third segment, whereas esophageal adenocarcinoma (EAC) typically develops from the metaplastic columnar epithelium in the lower third of the esophagus (5).

The survival rate of patients with esophageal cancer is inversely related to tumor invasiveness and the presence of nodal and distant disease (6). The 5-year overall survival (OS) for patients with early-stage disease after treatment exceeds $90 \%$, whereas the 5 -year OS is less than $40 \%$ for advanced-stage disease (6). Therefore, earlier detection allows for a better prognosis (6). However, because approximately $60-70 \%$ of patients who receive neoadjuvant chemoradiotherapy (nCRT) do not respond optimally, risk stratification and imaging biomarkers are used to dramatically improve the outcomes of treatment (6).

In the paradigm of precision medicine, diagnostic testing and targeted therapies are based on whole genome sequencing, as well as other clinical assessments and can have a significant clinical impact on the disease prognosis (7). In this review, we aim to assess the role of precision imaging in esophageal cancer, in terms of staging, restaging, biomarkers and radiomics, and its implications on disease prognosis.

\section{Pathogenesis and clinical picture}

The major risk factors of ESCC include tobacco and alcohol abuse, N-Nitrosamines, alkali burn, history of aerodigestive 
cancers, caustic ingestion and achalasia. The risk factors for EAC include chronic gastroesophageal reflux disease (GERD), smoking (2.18-fold increase), male sex (6-fold increase), obesity (2.39-increase), advancing age and Caucasian race $(4,8,9)$. Weekly GERD symptoms increase the risk of EAC by about 5 -fold with the risk increasing further in patients with chronic, nocturnal or more frequent symptoms (9). EAC can develop from progressive dysplastic changes within Barrett's esophagus. Barrett's esophagus develops secondary to reflux-induced metaplasia of the native squamous epithelium into intestinal epithelium (8). About $0.5 \%$ of patients with non-dysplastic Barrett's esophagus and $7 \%$ of patients with high-grade dysplasia will eventually progress to EAC (4).

Esophageal cancer is commonly diagnosed at an early stage incidentally during routine endoscopy or by surveillance of known Barrett's esophagus (15\% of EAC). However, most esophageal cancers are discovered when they have become locally advanced and are due to a non-specific initial presentation like heartburn or abdominal bloating. Therefore, as few as one out of eight esophageal cancers are detected at an early stage (T1) $(8,9)$. Esophageal cancer can present with dysphagia, vomiting, loss of body weight, fatigue and gastrointestinal bleeding $(8,9)$. Less commonly, it presents with oropharyngeal dysphagia or iron-deficiency anemia (9). Supra-clavicular lymph node (SCLN) metastasis can be diagnosed on clinical examination in approximately $8-20 \%$ of esophageal cancer cases (10).

\section{Genetics}

Esophageal cancer is associated with genetic mutations of the following genes: TP53, NOTCH, and MTOR; and amplification of the following genes: AKT2, EGFR, ERBB2 (HER2), FGFR1, KRAS, MDM2, and PIK3CA $(11,12)$. EAC is more commonly associated with HER2neu gene amplification and overexpression compared to ESCC (5). VEGFR expression is observed in 54\% of EAC patients and is correlated with poor survival (13). The wild-type p53 is a tumor suppressor in normal tissues that inhibits cell proliferation, whereas $\mathrm{p} 53$ overexpression is considered a potential tumor prognosticator $(14,15)$. Overexpression of p53 is seen in esophageal precancerous lesions and is involved in the stepwise progression of these lesions into esophageal cancer (14).

\section{Screening and diagnosis}

Upper endoscopy with biopsy and histopathological confirmation is the benchmark for diagnosis. Esophageal cancer can appear on endoscopy as a stricture, mass, raised nodule, ulceration, or a subtle irregularity in the mucosa (9).

Most observational studies have shown a survival benefit in the detection of EAC through Barrett's esophagus surveillance because increasing the possibility of identifying the disease in its early stages allows for the utilization of curative therapies. Multiple societies currently recommend regular endoscopic surveillance in Barrett's esophagus (16). Zhang and colleagues (14) found an increasing trend of the expression of carcinoembryonic antigen (CEA) and cancer antigen 19-9 (CA19-9) proteins with the progression from basal cell hyperplasia and esophageal dysplasia to invasive ESCC. A screening program to detect p53, CEA and CA19-9 proteins can help identify high-risk individuals with ESCC as they have a combined $84 \%$ specificity and $73 \%$ sensitivity for the diagnosis of ESCC (14).

\section{Radiological staging of esophageal cancer}

The 8th edition of the American Joint Committee on Cancer (AJCC) staging of epithelial cancers of the esophagus and esophagogastric junction (EGJ) is widely used today for initial staging (Table 1) (17). In order to ensure the best outcome and to determine the most suitable therapy, accurate radiologic staging between $\mathrm{T} 1$ and T2-4 is necessary. In addition, the large submucosal lymphatic network of the esophageal wall permits early regional nodal spread in esophageal cancer (18). A pilot study comparing the performance of different imaging modalities showed that for T staging, EUS had the best sensitivity and NPV (100\%), compared to magnetic resonance imaging (MRI) and MDCT (19). In addition, MRI also had the highest overall accuracy for $\mathrm{T}$ stage (83\%) (19). For nodal (N) staging, MRI and EUS had the highest sensitivity and NPV (100\%), however, MRI, EUS and MDCT did not show acceptable results for specificity and PPV (19) (Figure 1). For distant metastases (M) staging, Computed tomography (CT) and positron emission tomography/computed tomography (PET/CT) are the modalities of choice (4). 
Table 18 th edition of the American Joint Committee on Cancer (AJCC) staging of cancers of the esophagus and esophagogastric junction (EGJ)

\begin{tabular}{|c|c|}
\hline Category & Criteria \\
\hline TX & Tumor cannot be assessed \\
\hline TO & No evidence of primary tumor \\
\hline Tis & High-grade dysplasia, defined as malignant cells confined to the epithelium by the basement membrane \\
\hline T1a & Tumor invades the lamina propria or muscularis mucosae \\
\hline $\mathrm{T} 1 \mathrm{~b}$ & Tumor invades the submucosa \\
\hline T2 & Tumor invades the muscularis propria \\
\hline T3 & Tumor invades adventitia \\
\hline $\mathrm{T} 4 \mathrm{~b}$ & Tumor invades other adjacent structures, such as the aorta, vertebral body, or airway \\
\hline \multicolumn{2}{|c|}{$\mathrm{N}$ category } \\
\hline NX & Regional lymph nodes cannot be assessed \\
\hline NO & No regional lymph node metastasis \\
\hline N1 & Metastasis in one or two regional lymph nodes \\
\hline N2 & Metastasis in three to six regional lymph nodes \\
\hline N3 & Metastasis in seven or more regional lymph nodes \\
\hline \multicolumn{2}{|c|}{ M category } \\
\hline
\end{tabular}

\section{Endoscopic ultrasonography}

EUS can provide an accurate treatment assignment based on TNM staging in $75 \%$ of cases as compared to $65 \%$ with CT and $70 \%$ with PET/CT (20). EUS can help to determine the $\mathrm{T}$ stage by assessing the depth of invasion of the esophageal wall with $60-97 \%$ reported accuracy $(3,5)$. EUS also has better diagnostic performance in T4 disease ( $92 \%$ sensitivity and $97 \%$ specificity) than $\mathrm{T} 1$ disease (82\% sensitivity and $99 \%$ specificity) (21). The addition of EUS-guided fine needle aspiration biopsy to CT increases the sensitivity of $\mathrm{N}$-staging to $85-97 \%$ (5). EUS rarely identifies $M$ in remote sites because of its small field of view $(9,22)$, however, EUS combined with fine needle aspiration biopsy are very reliable in detecting $\mathrm{M}$ in non-regional nodes with $53 \%-98 \%$ sensitivity and $77-100 \%$ specificity (9). The limitations of EUS include invasiveness, operator dependency, difficulty with stenotic tumors, and risk of post-therapeutic inflammation and perforation $(3,5)$ (Figures 2,3).

\section{Multidetector $C T$}

MDCT plays an important role in the staging of esophageal cancer (22) with a reported accuracy of $43-92 \%$ in T-staging (23-26). MDCT cannot differentiate between the different layers of the esophageal wall and therefore, cannot readily distinguish between $\mathrm{T} 1$ and $\mathrm{T} 2$ tumors (22). T3 stage is detected on MDCT as periesophageal fat infiltration with $75 \%$ sensitivity and $78 \%$ specificity. T4 stage is identified with loss of fat planes between the tumor and adjacent mediastinal structure with $75 \%$ sensitivity and $86 \%$ specificity $(5,27)$. The diagnosis of T4 stage 

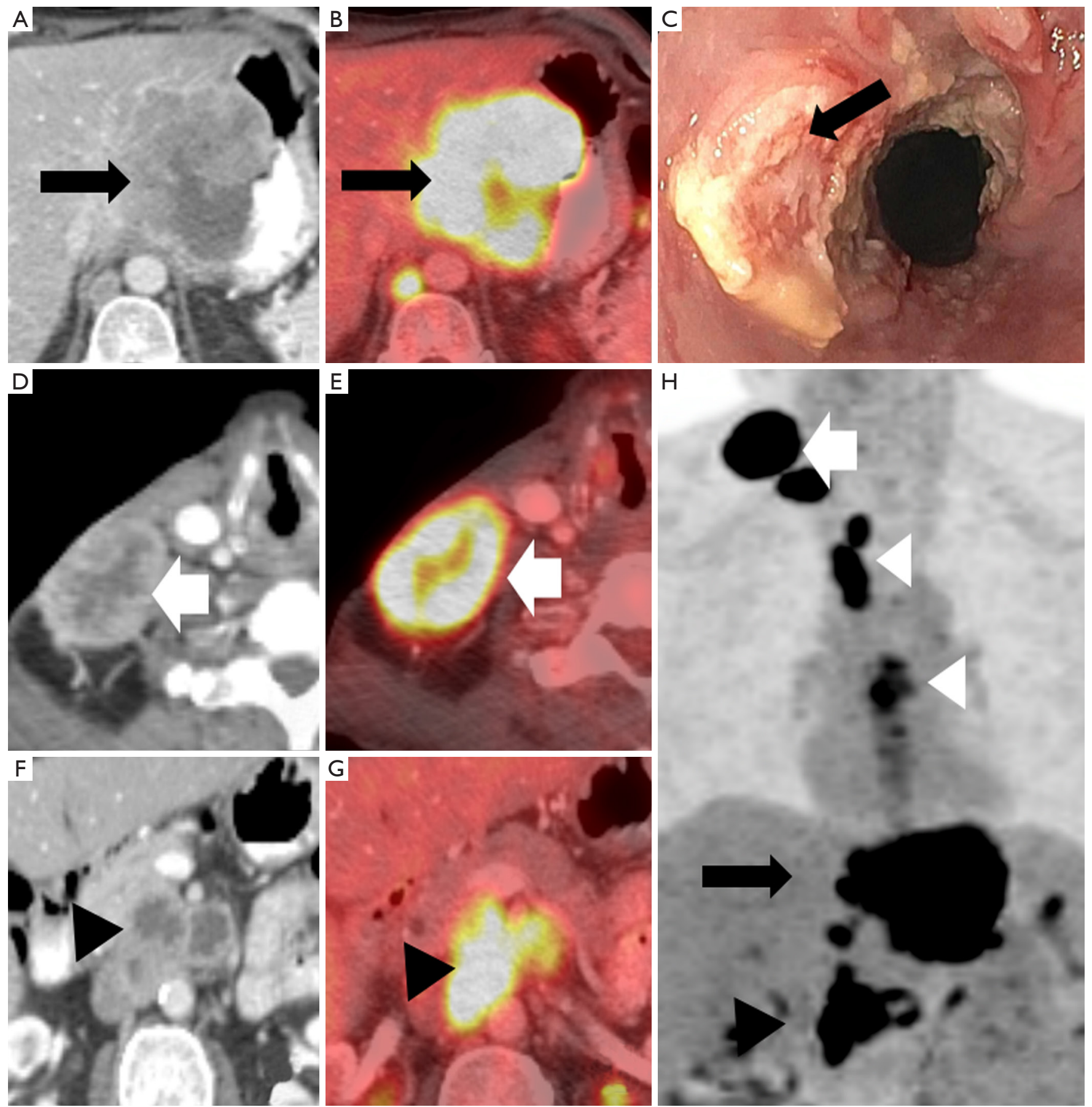

Figure 1 A 63-year-old male with squamous cell carcinoma of distal esophageal mass. (A) Axial contrast CT, (B) axial FDG PET/CT images show large necrotic FDG avid tumor at the lower esophagus (black arrow) invading the stomach, pancreas and liver. (C) EGD shows a circumferential partial obstructive ulcerative esophageal mass (black arrow). (D,F) Axial contrast CT and (E,G) axial FDG PET/CT images show FDG avid enlarged necrotic cervical (thick white arrows) (D,F) and retroperitoneal metastatic lymphadenopathy (black arrowheads) (E,G). (H) Coronal MIP image shows cervical (white arrow), mediastinal (white arrowhead) and retroperitoneal (black arrowhead) metastatic lymphadenopathy and distal esophageal carcinoma with metastatic lymphadenopathy (black arrow). PET, positron emission tomography; CT, computed tomography; EGD, esophagogastroduodenoscopy; MIP, Maximum Intensity Projection. 

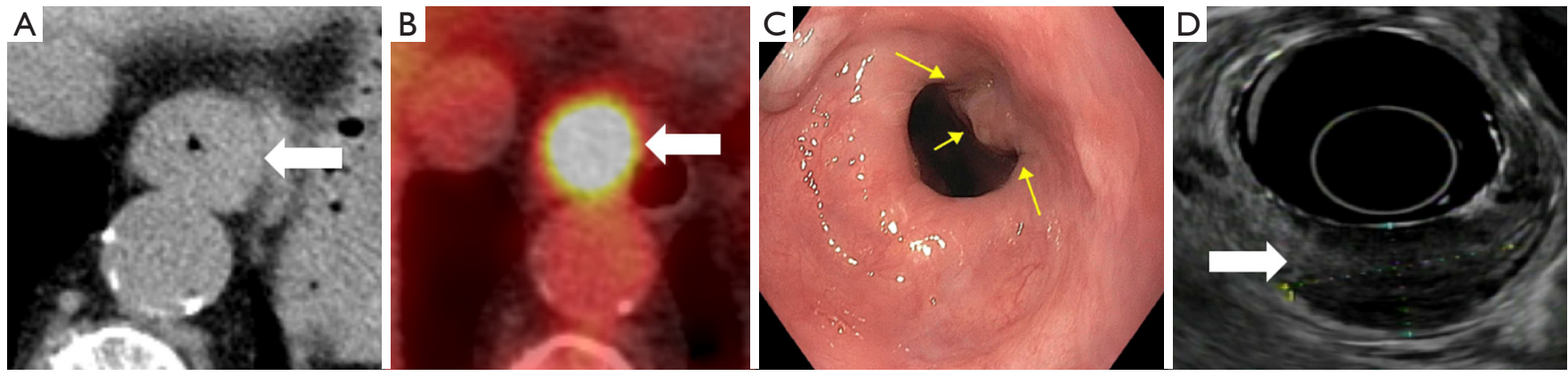

Figure 2 A 76-year-old female with adenocarcinoma of the gastroesophageal junction (GEJ). (A) Axial non-contrast CT and (B) axial FDG PET/CT images show asymmetrical irregular thickening FDG avid tumor (white arrow) at the GEJ invading the stomach, pancreas and liver. (C) EGD shows a single $2 \mathrm{~cm}$ mucosal nodule (yellow arrows) at GEJ. (D) EUS shows $2.0 \mathrm{~cm} \times 10 \mathrm{~cm}$ poorly defined onethird circumferential hypoechoic nodule/mass (white arrow) at the GEJ with likely invasion of muscularis propria. PET, positron emission tomography; CT, computed tomography; EGD, esophagogastroduodenoscopy; EUS, endoscopic ultrasound.
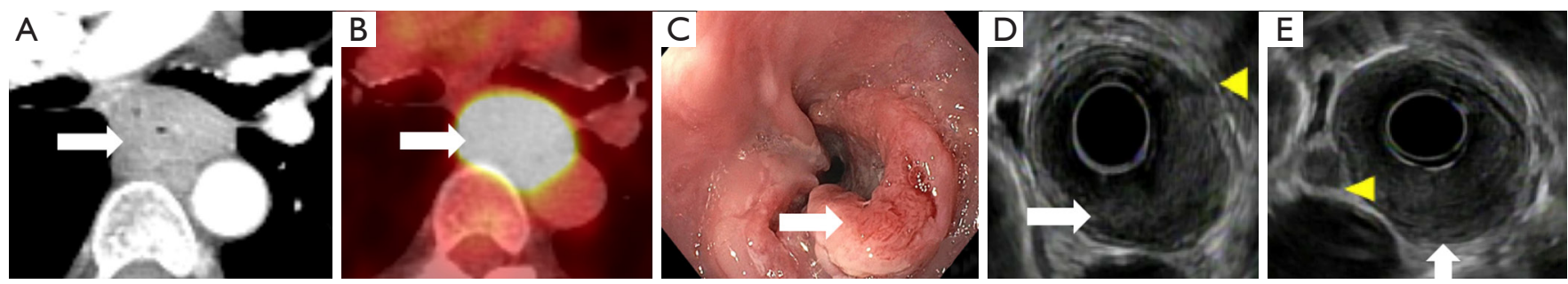

Figure 3 A 76-year-old female with squamous cell carcinoma at the mid esophagus. (A) Axial non-contrast CT and (B) axial FDG PET/ CT images show an FDG avid thickening at the mid esophagus (white arrow) abutting the posterior wall of the left mainstem bronchus. (C) EGD shows a friable exophytic mass in the mid esophagus (white arrow) with significant stenosis. (D,E) EUS shows near circumferential hypoechoic nodule/mass (white arrow) at the mid esophagus level with significant stenosis and penetration into the adventitia. A $0.7 \mathrm{~cm}$ $\times 0.5 \mathrm{~cm}$ hypoechoic peritumoral node (yellow arrowhead) consistent with metastatic invasion. PET, positron emission tomography; CT, computed tomography; EGD, esophagogastroduodenoscopy; EUS, endoscopic ultrasound.

can be challenging in patients who received surgery or radiotherapy and in cachectic patients secondary to the loss of fat planes (22). CT helps to detect aortic and tracheobronchial invasion with almost $100 \%$ sensitivity, albeit the specificity ranges from $52-97 \%$ (22).

The diagnostic role of CT in preoperative $\mathrm{N}$-staging in esophageal cancer is limited (18), with a reported $30-60 \%$ sensitivity, $60-80 \%$ specificity and $27-86 \%$ accuracy for nodes $>10 \mathrm{~mm}(5,23-26)$. It is difficult to establish a specific size threshold value to differentiate benign from malignant lymph nodes with high sensitivity and specificity at the same time. For example, Lie and colleagues (18) reported that lymph nodes $>10 \mathrm{~mm}$ could predict metastatic lymph nodes with a high PPV of $79 \%$, but only $28 \%$ sensitivity. By lowering the size diagnostic criterion the sensitivity improved to $37 \%$, but $63 \%$ of the metastatic nodes went undetected. In addition, normal-sized lymph nodes $(<10 \mathrm{~mm})$ could have microscopic metastatic foci that are usually undetected in CT resulting in understaging of the tumor $(18,28)$. Using criteria including size, shape and location of regional lymph nodes in esophageal cancer on CT could improve the staging sensitivity to $67 \%$ and the PPV of $64 \%$ compared with only short axis measurement in cT1 esophageal cancer $(18,28)$.

Contrast-enhanced CT (CECT) is the most commonly used modality for detecting $M$ in distant sites, particularly hepatic (Figure 4) and lung (Figure 5) metastases (29).

\section{PET/CT}

The PET/CT has a limited role in T staging of esophageal cancer other than affirming mediastinal organ invasion. However, it can help identify an occult primary neoplasm in patients presenting with metastatic disease (5). Fluorine-18 

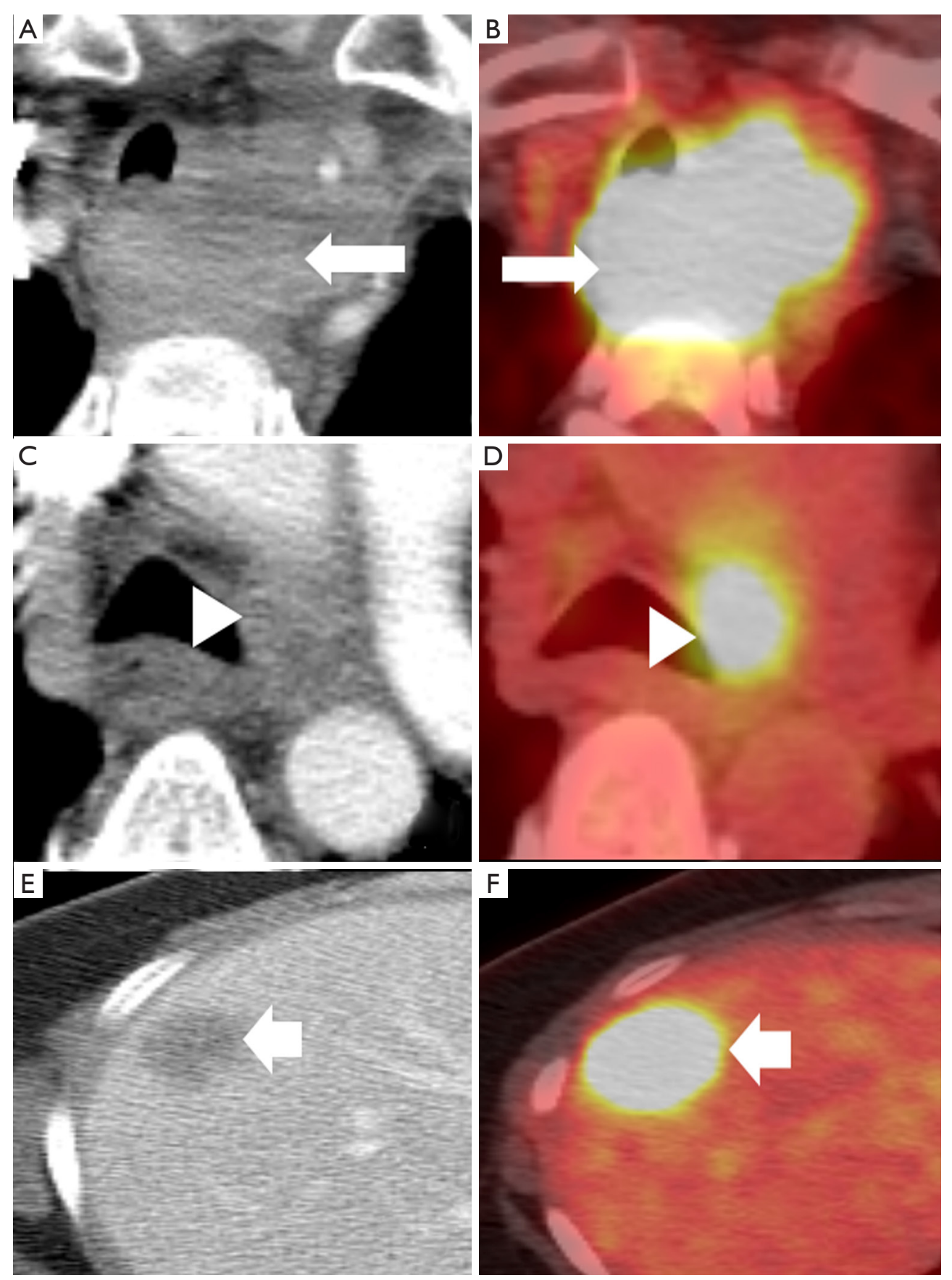

Figure 4 A 70-year-old female with squamous cell carcinoma at the proximal esophagus. (A,C,E) Axial non-contrast CT and (B,D,F) axial FDG PET/CT images shows FDG avid proximal esophageal mass (thin white arrows) (A,B) and right side displacement of the trachea (C,D) (white arrowheads) with FDG avid metastatic subcarinal lymph node and FDG avid liver metastasis (thick white arrows) (E,F). PET, positron emission tomography; CT, computed tomography. 

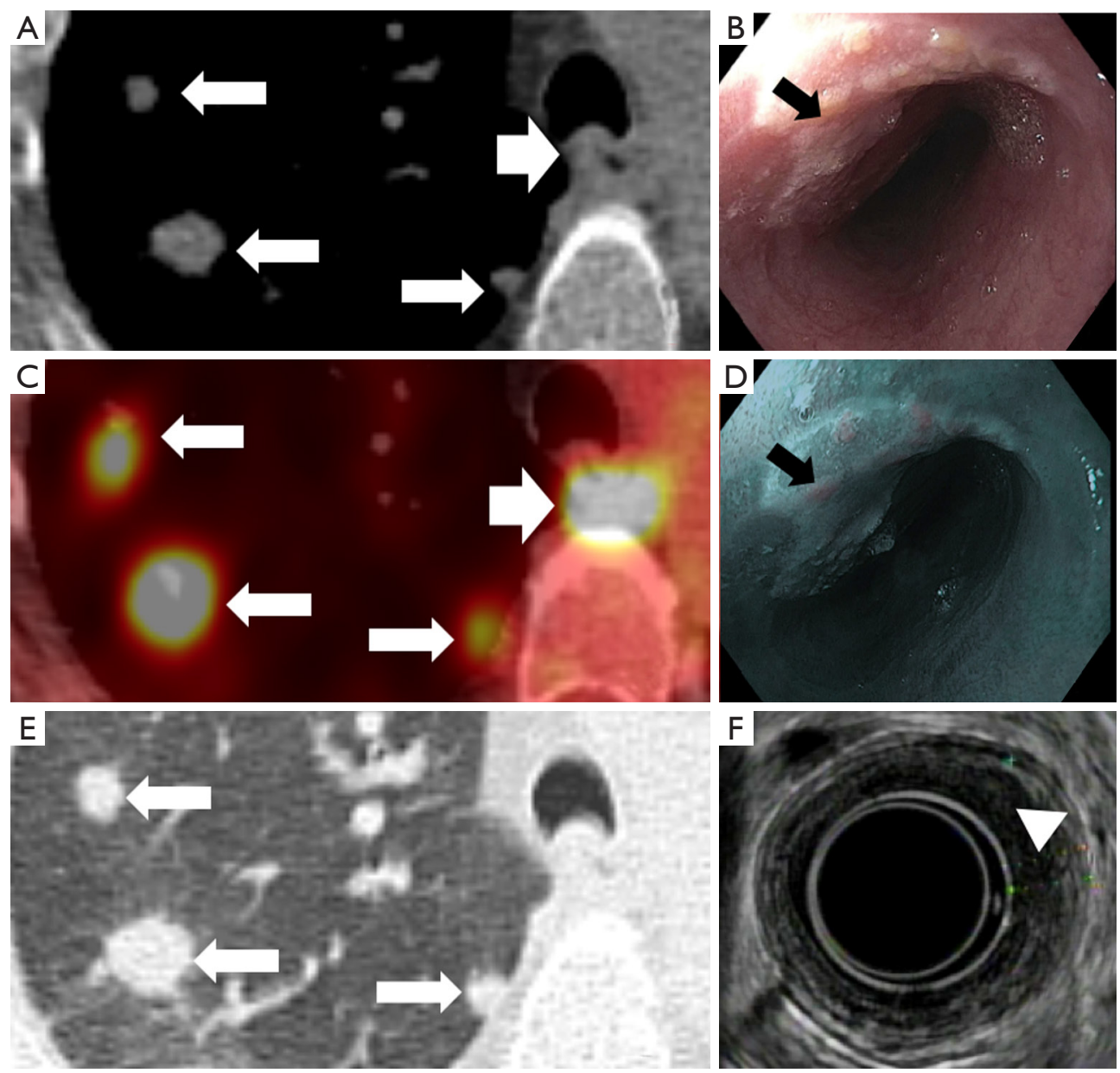

Figure 5 A 71-year-old male with squamous cell carcinoma at the mid esophagus. (A) Axial non-contrast CT soft tissue window, (C) axial FDG PET/CT and (E) axial non-contrast CT lung window shows mid esophageal mass (thick white arrow) and lung metastases (thin white arrows) and all are FDG avid in the FDG PET/CT image (C). (B,D) EGD shows nodular irregular mass at the mid esophagus (black arrow). (F) EUS shows an irregular hypoechoic ill thickening at the site of esophageal mass (white arrowhead) and it extends into submucosa with focal disruption of muscularis layer. PET, positron emission tomography; CT, computed tomography; EGD, esophagogastroduodenoscopy; EUS, endoscopic ultrasound.

fluorodeoxyglucose PET/CT $\left({ }^{18} \mathrm{~F}\right.$-FDG PET/CT) can assess the length and the volume of tumor in esophageal cancer patients who either cannot tolerate EUS or have impassable strictures (20-30\% of cases) (30).

The ${ }^{18}$ F-FDG PET/CT cannot accurately reflect the $\mathrm{N}$ status since the nodes are often obscured by the metabolic activity of the primary tumor or background activity $(5,31)$. A meta-analysis by Shi and colleagues (32) reported a pooled sensitivity and specificity of $62 \%$ and $96 \%$, per-station analysis, for ${ }^{18} \mathrm{~F}-\mathrm{FDG} \mathrm{PET} / \mathrm{CT}$ in the detection of nodal involvement (32). The high specificity of PET/CT allows nodal disease to be ruled out, preventing unnecessary surgeries (31). However, ${ }^{18} \mathrm{~F}$-FDG PET/CT is unable to detect microscopic metastasis and distinguish nodal metastatic disease from other benign conditions like reactive hyperplasia or granulomatous inflammation (28).

PET/CT is the modality of choice for detection of metastasis (M) with $71 \%$ sensitivity and $93 \%$ specificity (33). PET/CT has higher accuracy than either PET or CT alone in the diagnosis of $M(9)$. In a prospective trial of 129 patients, PET identified $41 \%$ additional metastatic sites, $38 \%$ had a shift in management and $8 \%$ detected synchronous malignancy (34).

\section{MRI}

In MRI, high-resolution T2-weighted imaging (T2WI) provides meticulous imaging of the anatomical layers of 

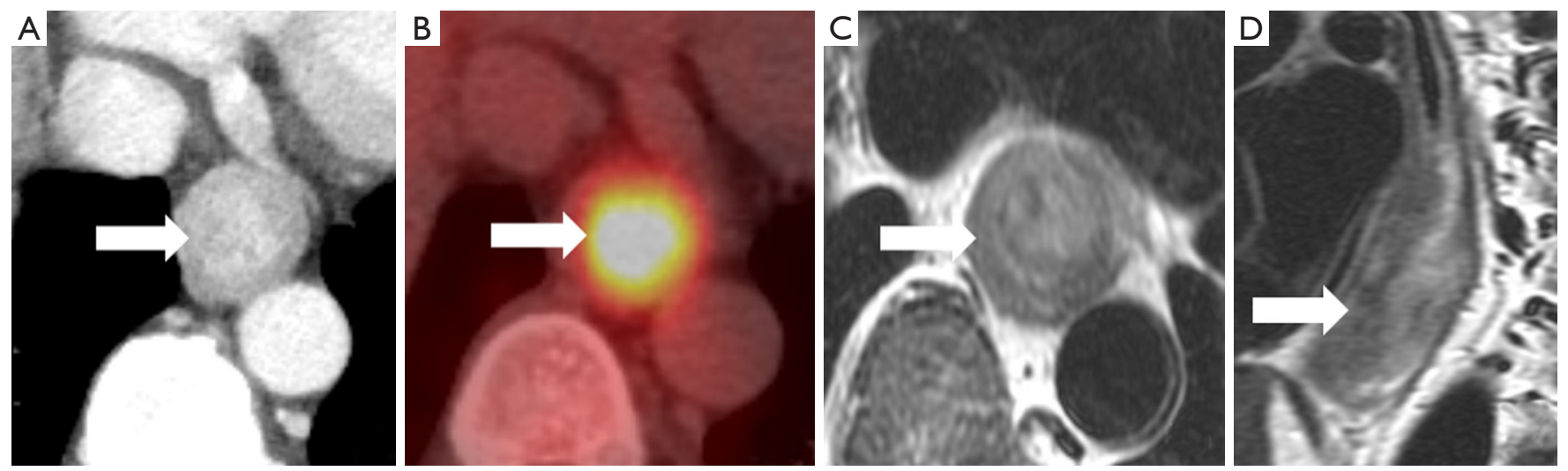

Figure 6 A 60-year-old male with adenocarcinoma of the GEJ. (A) Axial contrast CT, (B) axial FDG PET/CT, (C) axial T2WI and (D) coronal T2WI images show T2 isointense FDG avid irregular thickening (white arrows) at the GEJ. GEJ, gastroesophageal junction; PET, positron emission tomography; CT, computed tomography; T2WI, T2-weighted imaging.

the esophageal wall and surrounding tissues with an $81 \%$ accuracy for T-staging, but with a $16 \%$ understaging and $3 \%$ overstaging shortfall (35) (Figure 6). EAC appears isointense or slightly hyperintense on fat-suppression T2WI (FS-T2WI) and significantly hyperintense on diffusion-weighted-MRI (DW-MRI) (36). T2WI combined with DW-MRI can diagnose esophageal cancer with the detection rate of $33 \%$ for T1 stage, $58 \%$ for T2 stage, $96 \%$ for T3 stage and $100 \%$ for T4 stage (35). MRI showed comparable accuracy (75-87\%) to CT in assessing operability of esophageal cancer (35). The combination of DW-MRI and ${ }^{18} \mathrm{~F}$-FDG PET/CT provide complementary diagnostic and prognostic information in esophageal cancer since both increased metabolic activity and restricted water diffusion are independent parameters that reflect different aspects of tumor pathophysiology (37).

Conventional MRI shows moderately poor diagnostic performance for $\mathrm{N}$-staging in esophageal cancer with $25-62 \%$ sensitivity and $67-88 \%$ specificity (38). However, the use of a gadolinium contrast agent can improve the sensitivity and specificity of $1.5 \mathrm{~T}$ MRI scan up to $100 \%$ and $78 \%$, respectively, in the diagnosis of $\mathrm{N}$-staging (39). Also, Alper and colleagues (40) reported that MR imaging with STIR turbo spin-echo sequence predicted malignant lymph nodes in esophageal cancer with $81 \%$ sensitivity and $98 \%$ specificity. The increase in gross tumor volume of EAC acquired on FS-T2WI and DW-MRI with b-values of 500 and $800 \mathrm{~s} / \mathrm{mm}^{2}$ sequences is associated with a significant increase in the tumor N-stage (36). FS-T2WI is the best sequence for gross tumor volume measurement to differentiate stage N0 from stage N1-3 [0.806 area under the receiver operating curve (AUC), 74\% sensitivity and $92 \%$ specificity] (36)

The investigation of whole-body MRI (WBMRI) in esophageal cancer is still limited, however, WBMRI has an equivalent accuracy to ${ }^{18} \mathrm{~F}-\mathrm{FDG}-\mathrm{PET} / \mathrm{CT}$ in T-, N- and M-staging of esophageal cancer $(39,41)$. WBMRI also has the advantage of being used for serial follow-ups without the risk of overradiation that is encountered in PET/CT. In addition, SUV and ADC are entirely different tumor metrics that can complement each other (41).

Positron emission tomography/magnetic resonance imaging (PET/MRI) shows acceptable accuracy for $\mathrm{T}$ staging (67\%) compared with EUS (87\%) in esophageal cancer (42). In regards to N-staging, A higher sensitivity was noted in PET/MRI (83\%) compared to EUS $(75 \%)$, $\mathrm{PET} / \mathrm{CT}$ (67\%), and CT (50\%) (42). PET-MRI is also able to detect $M$ disease (29). PET/MRI has the advantage of superior soft tissue resolution and timesaving with the acquisition of both PET and MRI images simultaneously (29).

\section{Treatment}

The standard treatment for superficial T1 tumors is esophagectomy with endoscopic mucosal resection or ablation for mucosal tumors $(5,22)$. Concurrent chemoradiotherapy (CRT) should be considered for cervical and upper thoracic esophageal cancer (5). Locally advanced esophageal cancer is managed with nCRT, definitive chemoradiotherapy (dCRT), perioperative chemotherapy (for EAC), or esophagectomy $(5,43)$. The use of nCRT in locally advanced esophageal 

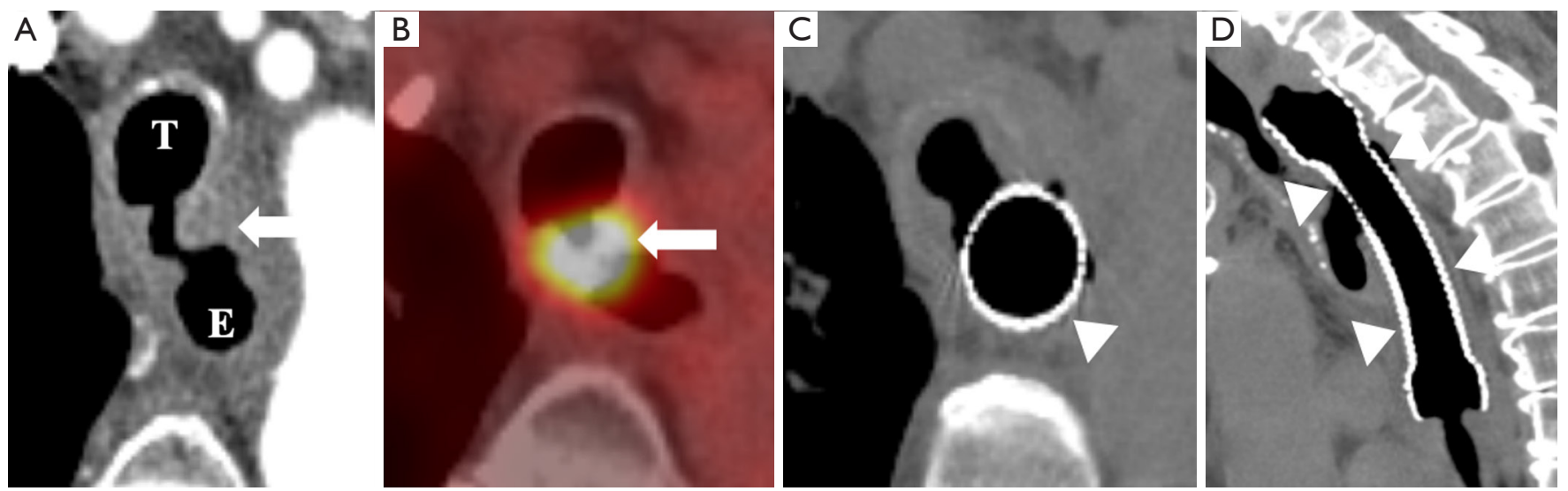

Figure 7 A 59-year-old female with squamous cell carcinoma at the proximal esophagus. (A) Axial contrast CT and (B) axial FDG PET/ CT images show an FDG avid $3.7 \mathrm{~cm}$ thickening in the proximal esophagus (thin white arrows) and tracheoesophageal fistula. (C) Axial and (D) coronal non-contrast CT shows an esophageal stent (white arrowheads) extending from thoracic inlet up to the subcarinal level. PET, positron emission tomography; CT, computed tomography; T, trachea; E, esophagus.

cancer is associated with improved OS and pathological complete response (pCR) (44). The most commonly used two-drug chemotherapy is 5 -FU and cisplatin with the addition of docetaxel or epirubicin reserved for good responders (5). The decision as what which adjuvant treatment to use following nCRT or dCRT is based on response assessment on imaging, usually PET/CT, or endoscopy; esophagectomy or observation for patients with complete response (CR); salvage esophagectomy or palliative therapy for non-responders (5) (Figure 7).

Stage IV disease is treated with palliative radiotherapy and chemotherapy (22). Anti-HER2 monoclonal antibodies (Trastuzumab) are recommended in advanced or metastatic EAC overexpressing HER2-neu receptors (5). Multiple targeted therapies including VEGF/VEGFR inhibitors (such as Bevacizumab and Ramucirumab) and EGFR tyrosine kinase inhibitors (such as erlotinib and gefitinib) are under research for advanced and metastatic esophageal cancer $(5,12,13)$. The genetic profile of ESCC provides a foundation for potential novel targeted therapies and precision medicine (11).

\section{Restaging and the role of imaging in the assessment of treatment response}

The local recurrence in ESCC patients is the primary reason for failure after CRT and is associated with poor prognosis. Hence, the accurate assessment of the tumor response to CRT is crucial for predicting survival and guiding the management (43) (Figure 8). Currently, there is no optimum guideline available to inform practice (45). The National Comprehensive Cancer Network (NCCN) advises restaging for all patients who receive nCRT (46), whereas the European Society for Medical Oncology (ESMO) recommends restaging of patients with cT3-4 or cN1-3 tumors (47).

\section{$C T$}

CT has $33-55 \%$ sensitivity and $50-71 \%$ specificity in the assessment of tumor response after nCRT $(43,48)$. The change in tumor volume calculated on CT scans has a limited role in predicting pathological response to nCRT in esophageal cancer patients $(0.742$ AUC, $56 \%$ sensitivity, $93 \%$ specificity, $\mathrm{P}=0.005$ ) (49). The CT number of a tumor may be useful to assess treatment response in advanced esophageal cancer. A threshold value for tumor CT number of $>40$ Hounsfield Units can predict encouraging response to chemotherapy with 0.73 AUC (50).

CT perfusion parameters in the esophageal wall affected by the tumor display a strong positive correlation with the histopathologic tumor regression grade according to Mandard's criteria (51). The median blood flow and blood volume are gradually increased, while the mean transit time is decreased as tumor regression grade is increased secondary to an increase in the neovascularization with higher tumor grades (51). Also, CT perfusion values can detect the hypervascularized residual viable tumor in the esophageal wall in incomplete or non-responders to nCRT (51). 

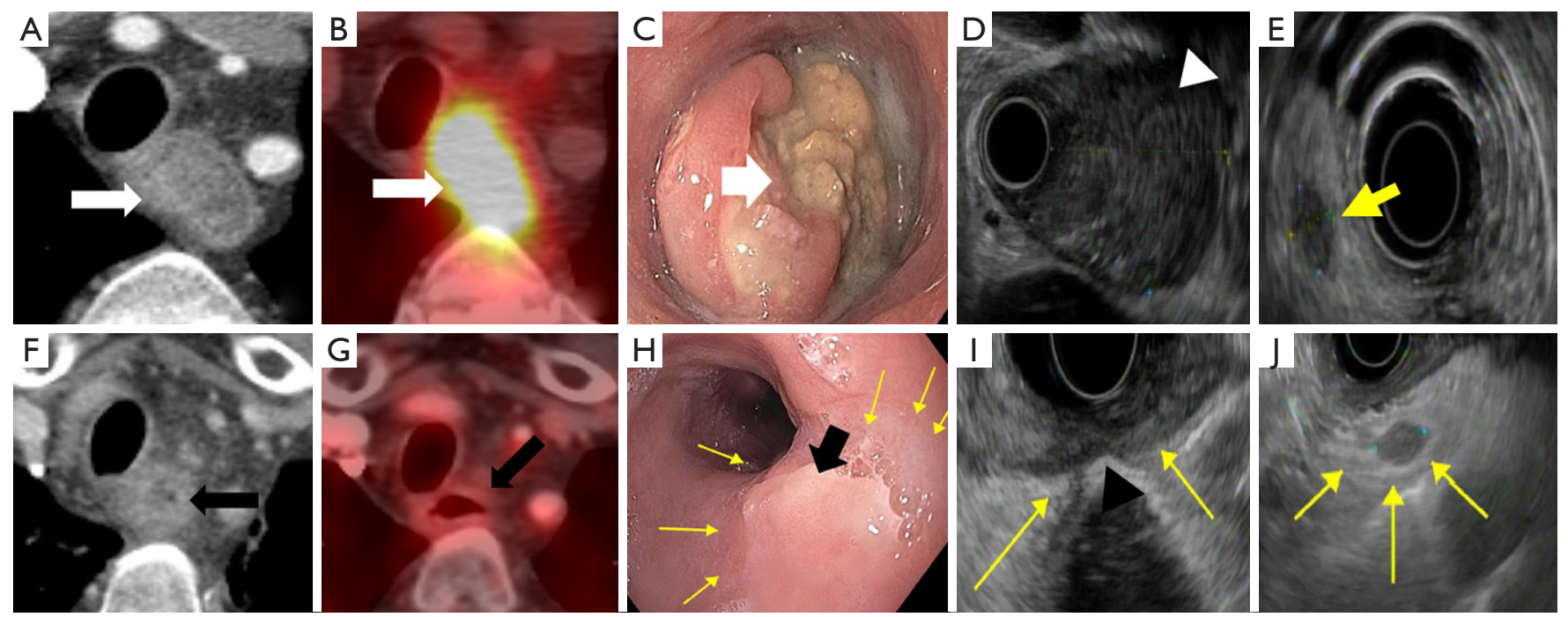

Figure 8 A 57-year-old male with squamous cell carcinoma at the proximal esophagus. (A) Axial contrast CT and (B) axial FDG PET/ CT images show an FDG avid proximal esophageal mass (thin white arrow); (C) EGD shows a large ulcerative partially obstructive noncircumferential mass (thick white arrow) in the upper one-third of the esophagus; (D,E) EUS shows a $3 \mathrm{~cm}$ thickness of the wall of the esophagus (white arrowhead) and a $1 \mathrm{~cm}$ oval hypoechoic well defined right paraesophageal lymph node (thick yellow arrow). He underwent 3 months of chemoradiation. (F) Axial contrast CT and (G) axial FDG PET/CT images show interval decrease in the size and FDG activity of the proximal esophageal mass (thick black arrow). (H) EGD shows mild residual thickening in the upper one-third of the esophagus (thick black arrow) with surrounding normal esophageal mucosa (thin yellow arrows). (I,J) EUS shows a mild residual thickening thickness of the wall of the esophagus (black arrowhead) and reduction in $0.5 \mathrm{~cm}$ oval hypoechoic right paraesophageal lymph node (thin yellow arrows). PET, positron emission tomography; CT, computed tomography; EGD, esophagogastroduodenoscopy; EUS, endoscopic ultrasound.

Using dual-source dual-energy CT (DECT) iodine map, the changes of normalized iodine concentration (NIC) before and after CRT in esophageal cancer patients can monitor the response to CRT. The responders have significantly lower NIC in both hepatic arterial and portal venous phases compared to the non-responders (52). The reduced tumor iodine intake is due to the reduced vascular proliferation and supply of esophageal cancer secondary to CRT treatment (52).

\section{PET/CT}

A recent meta-analysis that included 13 studies $(697$ patients) to determine the role of ${ }^{18} \mathrm{~F}$-FDG-PET/CT $\left(\mathrm{PET}_{\mathrm{per}}\right)$ in the assessment of the response to CRT in esophageal cancer concluded that the role of $\mathrm{PET}_{\text {per }}$ in esophageal cancer patients is controversial and its predictive and prognostic value cannot be definitively established (53). In particular, 8 studies supported the predictivity of $\mathrm{PET}_{\text {per }}$, whereas 5 did not find any correlation between $\mathrm{PET}_{\text {per }}$ parameters, the pCR and/or the clinical outcome. PET $\mathrm{P}_{\text {per }}$ predicted pCR with $63-100 \%$ sensitivity, 50-76\% specificity and 51-89\% AUC; OS with 49-94\% sensitivity, 36-82\% specificity and 61-93\% AUC and the DMFS/progression-free survival (PFS) with 7794\% sensitivity, 79-82\% specificity and 57-93\% AUC (53).

One study reported that pCR in primary tumors was associated with post-treatment maximum standard uptake value (SUVmax) values $(\mathrm{P}=0.016)$ and percent change in intratumoral SUVmax $(\mathrm{P}=0.006)(44)$. Posttreatment SUVmax cutoff value of $\leq 3.25$ predicted $\mathrm{pCR}$ with 0.752 AUC, $67 \%$ sensitivity and $67 \%$ specificity; and post-treatment percent change in SUVmax cutoff value of $>72.32 \%$ predicted pCR with 0.705 AUC, $71 \%$ sensitivity and $67 \%$ specificity (44). Another study by Zschaeck and colleagues (54) in locally advanced esophageal cancer reported an increase in SUVmax and SUVmean in the non-tumor-affected esophagus on restaging PET was significantly associated with improved OS, better local control, and a lower rate of treatment failure or the development of distant metastases (54). Findlay and colleagues (55) found that the composite 
measurement of FDG-avidity and volume metrics can improve the prediction of treatment response, as $\Delta \mathrm{SUVmax}$ and $\Delta$ length of the tumor are independent predictors of pCR (55). In addition, metabolic nodal response, which is often discordant with the primary tumor response, can specifically assess metastatic deposits that are likely responsible for recurrence (55). Distant interval metastases have been reported to be detected on $0-26 \%$ of esophageal cancer patients on presurgical restaging after nCRT. It is crucial to identify these patients prior to surgery because esophagectomy carries a high morbidity rate; additional systemic therapy could be considered in these patients (56) However, ${ }^{18} \mathrm{~F}-\mathrm{FDG}$ PET/CT can result in false positive results in $5 \%$ of patients, indicating the necessity for the histopathological confirmation of suspected lesions (56).

Tamandl and colleagues (3) proposed an algorithm of combined CECT and ${ }^{18} \mathrm{~F}-\mathrm{FDG}$ PET/CT for locoregional restaging of esophageal cancer after nCRT and it was able to determine post-therapeutic $\mathrm{T}$ stage with a sensitivity, PPV, and accuracy of $83 \%, 91 \%$, and $76 \%$ compared to $79 \%, 70 \%$, and $59 \%$ in CECT, and $81 \%, 81 \%$, and $68 \%$ in visual ${ }^{18} \mathrm{~F}$-FDG PET/CT, respectively. The combined CECT and MTV had the highest diagnostic accuracy to predict CR (0.82 AUC, $\mathrm{P}<0.001)$.

\section{MRI}

In regards to DW-MRI in esophageal cancer, responders to nCRT have a lower baseline and higher post-treatment ADC values compared to non-responders $(19,57)$. One could speculate that the non-responders have necrotic areas within the tumor accounting for the higher pre-treatment ADC values and decreasing the response to nCRT (19). Conversely, an alternate study reported that high baseline ADC values correlated well with better response to CRT and higher survival rates in esophageal cancer patients (58). A low percent change in tumor ADC during the first 2-3 weeks of nCRT $\left(\triangle \mathrm{ADC}_{\mathrm{per}}\right)$ indicates a low rate of cell membrane integrity loss during treatment and $\triangle \mathrm{ADC}_{\text {per }}$ of $<29 \%$ was predictive of residual cancer with $100 \%$ sensitivity, $75 \%$ specificity, 94\% PPV, and 100\% NPV (59). Also, a low $\triangle \mathrm{ADC}_{\text {per }}$ of $<21 \%$ was predictive of no-pCR with $82 \%$ sensitivity, $100 \%$ specificity, $100 \% \mathrm{PPV}$, and $80 \% \mathrm{NPV}$ and these findings can guide the early management (59). One of the drawbacks of DW-MRI is being insensitive to inflammation early during treatment, however, this might be overcome by the addition of ${ }^{18} \mathrm{~F}$-FDG-PET/CT (59).

The DCE-MRI parameters can also help predict and monitor response to concurrent CRT for advanced esophageal cancer since the $\mathrm{K}^{\text {trans }}$ and $\mathrm{K}_{\mathrm{ep}}$ values are closely correlated with the degree of tumor microcirculation and angiogenesis (60). The CR is associated with higher baseline $\mathrm{K}^{\text {trans }}$ and $\mathrm{K}_{\mathrm{ep}}$ values; lower posttreatment $\mathrm{K}^{\text {trans }}$ and $\mathrm{K}_{\mathrm{ep}}$ values; and higher absolute change and ratio of change of both $\mathrm{K}^{\text {trans }}$ and $\mathrm{K}_{\mathrm{ep}}$. Among the pre-CRT parameters, high pre- $\mathrm{K}^{\text {trans }}$ value is the best parameter because it is associated with excellent treatment response, due to better blood perfusion, delivery of chemotherapy and higher radiosensitivity. For post-CRT measurements, post- $\mathrm{K}_{\mathrm{ep}}$ is the best parameter to assess treatment response with a threshold value of $<1.031$ predicts CR with $95 \%$ sensitivity, $57 \%$ specificity and $0.817 \mathrm{AUC}(60)$. In terms of change of the DCE-MRI parameters, the $\Delta \mathrm{K}^{\text {trans }}$ is the best parameter to assess treatment response with an optimal threshold of $>-0.206$ predicts $\mathrm{CR}$ with $53 \%$ sensitivity, $95 \%$ specificity and $0.816 \mathrm{AUC}(60)$. The best parameter to assess the ratio of change of DCE-MRI is the ratio of $\Delta \mathrm{K}^{\text {trans }}$ with an optimal threshold of $>-0.144$ predicts CR with $90 \%$ sensitivity, 62\% specificity and 0.840 AUC (60).

Heethuis and colleagues reported that the combination of both DW-MRI and DCE-MRI can provide complementary information in predicting the response to nCRT in esophageal cancer, resulting in a high predictive value, that is higher than ${ }^{18} \mathrm{~F}$-FDG PET(/CT) (c-index $\left.=0.89\right)(61)$.

\section{Radiogenomics and radiomics}

The differences in genetic drivers between tumors can be assessed by their FDG avidity. FDG uptake is a surrogate for glucose metabolism in the tumor. Elevated FDGuptake in operable EAC correlates with multiple oncogenic processes with potential therapeutic targets (62). For instance, Heiden and colleagues (62) reported that FDGavid tumors were associated with increased expression of multiple matrix metalloproteinases, extracellular matrix components, members of oncogenic signaling pathways, and $\mathrm{PD}-\mathrm{L} 1$ proteins (fold change $>2.0, \mathrm{P}<0.05$ ). In addition, FDG-avid tumors had significant upregulation of specific gene sets associated with extracellular matrix organization (metastasis) and vascular development (angiogenesis) $(\mathrm{P}<0.005)(62) . F D G$-avid tumors are associated with the signaling pathways of hypoxia, angiogenesis, KRAS signaling, and epithelial-to-mesenchymal transition; and tumors with low FDG uptake were associated with increased gene sets of oxidative phosphorylation and MYC signaling (62). 
KRAS plays an important role in the PI3K and Raf/ MEK/ERK signaling pathways regulating cell metabolism and subsequently esophageal cancer tumorigenesis. These aforementioned oncogenic pathways represent aggressive tumor phenotypes, and thus esophageal cancer might benefit from neoadjuvant treatments targeting these pathways (62). Surprisingly, KRAS expression was not associated with a worse prognosis overall $(\mathrm{P}=0.64)(62)$. Development of a predictive imaging model based on these molecular factors may establish a relationship between KRAS and the other molecular alterations of esophageal cancer, allowing for enhanced precision management of esophageal cancer.

Tan and colleagues found that a CT-based radiomic model can predict LN metastasis in ESCC patients and outperforms size criteria with 0.758 AUC in a training set, and $0.773 \mathrm{AUC}$ in the validation set (63). CT radiomic signature extracted from baseline CT scans might aid in distinguishing CR from partial response in liver metastases from esophageal cancer (64-68). Hou and colleagues (65) found that a radiomic prediction model including histogram skewness, histogram kurtosis, gray-level size-zone matrix (GLSZM) long-zone emphasis, and 2 Gabor transformed parameters MSA-54 and MSE-54, differentiated nonresponders from responders to $\mathrm{nCRT}$ in esophageal cancer (65). A retrospective study of 18 patients reported that a CT based radiomic model can predict a significant decrease in volume of liver metastases to chemotherapy with 0.64 AUC for partial response lesions and 0.79 AUC for CR lesions (64). Larue and colleagues (69) proposed a pretreatment CT-based radiomic model that was able to stratify patients into statistically significant risk groups and also predicted a 3 -year OS in esophageal cancer patients with better prognostic performance compared to the clinical model (69). In addition, lower baseline histogram uniformity on unenhanced CT images and higher posttherapeutic entropy on CECT were associated with poor OS $(66,67)$.

Multiple ${ }^{18} \mathrm{~F}$-FDG PET studies found that various first, second and high-order features differentiated responders and non-responders to nCRT and also predicted pCR in esophageal cancer (68,70-77). Tixier and colleagues (70) showed that, in 41 patients, gray-level co-occurrence matrix (GLCM) homogeneity, GLCM entropy, GLSZM sizezone variability, and run length matrix intensity variability differentiated non-responders, partial response, and CR with $76-92 \%$ sensitivity (70). Another study reported that the ${ }^{18}$ F-FDG PET-derived textural feature 'long run low gray level emphasis' and CT-derived textural feature 'run percentage' were more accurate in assessing the response of esophageal cancer to nCRT than SUVmax (71). Foley and colleagues (74) proposed a prognostic model of three PET texture metrics, $\log$ (TLG), $\log$ (histogram energy) and histogram kurtosis, which are significantly and independently associated with OS $(\mathrm{P}<0.001)(74)$. According to the model, tumors with increased $\log$ (TLG) and histogram kurtosis, and reduced $\log$ (histogram energy) have an increased likelihood of mortality (74).

In addition, MRI radiomic features might be able to predict metastatic nodal disease in esophageal cancer patients. One study identified a radiomic MR model of nine radiomic features extracted from MR images (T2-TSEBLADE and contrast-enhanced StarVIBE) and this model was significantly associated with $\mathrm{LN}$ metastasis $(\mathrm{P}<0.001)$ and differentiated metastatic and non-metastatic lymph nodes with $0.821 \mathrm{AUC}$ in the primary cohort and 0.762 AUC in the validation cohort (78).

\section{Recurrent disease}

There is a high incidence of post-therapeutic recurrence of $45-53 \%$ in esophageal cancer and most recurrences occur within the first two years after surgery with a median time to recurrence of $10-12$ months $(79,80)$. Routine imaging with CT and PET/CT has been effective in the systematic follow-up of asymptomatic patients and early detection of recurrence (81-84) (Figure 9). However, there has been no established protocol for a follow-up yet (84).

A meta-analysis by Goense and colleagues (81) showed that ${ }^{18} \mathrm{~F}$-FDG PET and PET/CT have pooled estimates of sensitivity and specificity of $89-100 \%$ and $55-94 \%$, respectively, in the diagnosis of recurrent esophageal cancer after treatment with curative intent. There was no significant difference in diagnostic accuracy between PET and integrated PET/CT $(\mathrm{P}=0.213)(81)$. In the diagnosis of recurrent esophageal cancer, compared to ${ }^{18} \mathrm{~F}-\mathrm{FDG}$ PET and PET/CT, CT has a lower sensitivity (65-89\%) secondary to the misdiagnosis of postoperative changes and scarring; and higher specificity (79-91\%) because of the false-positive FDG avidity in the gastric tube and thoracic lymph nodes $(35,82,83)$. Therefore, integrated PET/CT seems to be the most accurate modality for the detection of recurrent esophageal cancer (35). PET/CT has the advantage of scanning the whole body and the ability to detect small lymph node metastases up to $0.9 \mathrm{~mm}$ diameter as well as recurrent tumors outside the body $(84,85)$. 

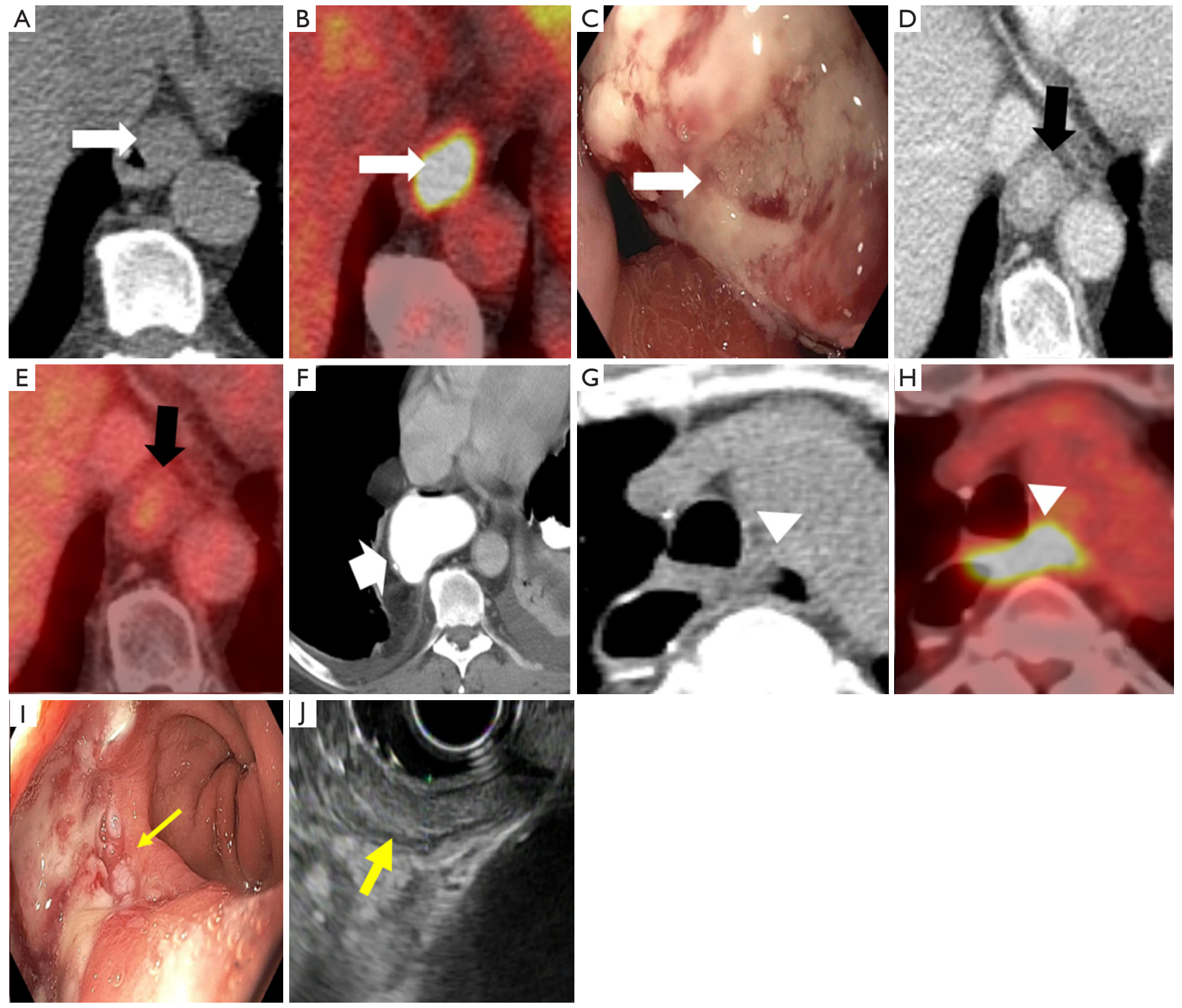

Figure 9 A 63-year-old male with adenocarcinoma of a distal esophageal mass. (A) Axial non-contrast CT and (B) axial FDG PET/CT images show an FDG avid thickening of the distal esophagus (white arrow). (C) EGD shows a ulcerative distal esophageal mass (thick white arrow). After chemoradiation, (D) axial contrast CT and (E) axial FDG PET/CT images show interval decrease in FDG avidity and thickening at the distal esophagus (black arrows). (F) Axial CT image with oral contrast shows the gastric conduit (thick short white arrow) after the patient underwent Ivor-Lewis esophagectomy. A follow-up CT after 1 year (G) axial non-contrast CT and (H) axial FDG PET/ CT images show focal FDG uptake with a circumferential thickening at the anastomosis the native esophagus and interposed conduit (white arrowheads). (I) EGD shows a non-obstructing non-circumferential fungating mass in the upper esophagus (thin yellow arrow). (J) EUS shows a $1.3 \mathrm{~cm}$ localized wall thickening in the upper esophagus (thick yellow arrow) with focal disruption of muscularis propria without lymphadenopathy. PET, positron emission tomography; CT, computed tomography.

The available literature on the role of MRI for the detection of recurrent esophageal cancer is scarce. However, Kantarci and colleagues (86) reported that T2WI MRI has higher diagnostic accuracy than CT in the evaluation of wall thickening and the diagnosis of osseous metastases. Another study reported that recurrent nodal disease shows evident diffusion restriction with $81 \%$ accuracy and that a cut-off apparent diffusion coefficient (ADC) value of $<1.5$ diagnoses recurrent nodal disease with $100 \%$ overall accuracy $(87)$. 


\section{Prognosis}

The overall 5-year survival rates of patients with esophageal cancer who are treated with curative intent are relatively poor (34-47\%) (81). EAC has a better overall 5-year survival rate (47\%) than ESCC (37\%) (22). Esophageal cancer with NOTCH1 mutation and EGFR genetic mutations are associated with larger tumor size $(\mathrm{P}=0.019)$ and lesser invasion depth $(\mathrm{P}=0.005)$ (11) and EGFR gene may be used as a clinically valuable biomarker to predict the prognosis of ESCC patients (12). Okumura and colleagues (88) reported statistically significant correlations between $\mathrm{p} 53$ expression and a favorable response to CRT.

Sequential ${ }^{18} \mathrm{~F}$-FDG PET/CT metabolic parameters of the primary tumor can monitor the response to CRT and reflect the OS and recurrence-free survival of esophageal cancer patients and thus guide clinical decisions in patient management $(89,90)$. Lymph node involvement bears an important prognostic value for survival in esophageal cancer $(31,91)$. Xu and colleagues (10) reported that patients with SCLN metastasis had a worse OS, DMFS and PFS $(\mathrm{P}<0.001)$ than those without SCLN metastasis (10). Baseline TLG $(\mathrm{P}=0.002)$ and SUVmax $(\mathrm{P}=0.003)$ of nodal metastasis at PET/CT can independently predict OS and recurrence-free survival (89). Increased recurrence in ESCC was independently associated with the presence of extracapsular extension $(24 \%$ vs. $69 \%, \mathrm{P}<0.001)$, lymphovascular invasion $(18 \%$ vs. $69 \%, \mathrm{P}=0.006)$ and nodal positivity ( $41 \%$ vs. $71 \%, \mathrm{P}=0.041)$ (44).

\section{Conclusions}

Imaging plays a pivotal role in staging, risk stratification and selection of therapeutic strategies. The imaging modalitiesCT, EUS, PET/CT and MRI, are usually complementary in the staging of esophageal cancer. EUS is the modality of choice for T staging, while CT and PET/CT are most effective at detecting metastasis. For $\mathrm{N}$ staging, MRI and EUS have the highest sensitivity, while all the other imaging modalities have comparable specificity. The assessment of treatment response with different imaging modalities in esophageal cancer provides significant prognostic information necessary to construct a treatment plan consisting of effective and efficient therapeutic measures.

\section{Acknowledgments}

Funding: None.

\section{Footnote}

Provenance and Peer Review: This article was commissioned by the Guest Editor (Ammar Chaudhry) for the series "Role of Precision Imaging in Thoracic Disease" published in Fournal of Thoracic Disease. The article was sent for external peer review organized by the Guest Editor and the editorial office.

Conflicts of Interest: All authors have completed the ICMJE uniform disclosure form (available at http://dx.doi. org/10.21037/jtd.2019.08.15). The series "Role of Precision Imaging in Thoracic Disease" was commissioned by the editorial office without any funding or sponsorship. The authors have no other conflicts of interest to declare.

Ethical Statement: The authors are accountable for all aspects of the work in ensuring that questions related to the accuracy or integrity of any part of the work are appropriately investigated and resolved.

Open Access Statement: This is an Open Access article distributed in accordance with the Creative Commons Attribution-NonCommercial-NoDerivs 4.0 International License (CC BY-NC-ND 4.0), which permits the noncommercial replication and distribution of the article with the strict proviso that no changes or edits are made and the original work is properly cited (including links to both the formal publication through the relevant DOI and the license). See: https://creativecommons.org/licenses/by-nc-nd/4.0/.

\section{References}

1. Herszényi L, Tulassay Z. Epidemiology of gastrointestinal and liver tumors. Eur Rev Med Pharmacol Sci 2010;14:249-58.

2. Sengupta N, Sawhney MS. Advances in imaging and endoluminal therapies for early esophageal and gastric cancers. Ann Surg Oncol 2016;23:3774-9.

3. Tamandl D, Fueger B, Haug A, et al. A diagnostic algorithm that combines quantitative $18 \mathrm{~F}-\mathrm{FDG}$ PET parameters and contrast-enhanced ct improves posttherapeutic locoregional restaging and prognostication of survival in patients with esophageal cancer. Clin Nucl Med 2019;44:e13-e21.

4. Mkarimi M, Mashimo H. Advanced imaging for Barrett's esophagus and early neoplasia: surface and subsurface imaging for diagnosis and management. Curr 
Gastroenterol Rep 2018;20:54.

5. Tirumani H, Rosenthal MH, Tirumani SH, et al. Esophageal carcinoma: current concepts in the role of imaging in staging and management. Can Assoc Radiol J 2015;66:130-9.

6. Uemura N, Kondo T. Current advances in esophageal cancer proteomics. Biochim Biophys Acta 2015;1854:687-95.

7. Triadafilopoulos G, Friedland S. Precision care for Barrett's esophagus. Transl Gastroenterol Hepatol 2018;3:67.

8. Meves V, Behrens A, Pohl J. Diagnostics and early diagnosis of esophageal cancer. Viszeralmedizin 2015;31:315-8.

9. Rubenstein JH, Shaheen NJ. Epidemiology, diagnosis, and management of esophageal adenocarcinoma. Gastroenterology 2015;149:302-17.e1.

10. Xu HY, Wu SX, Luo HS, et al. Analysis of definitive chemo-radiotherapy for esophageal cancer with supraclavicular node metastasis based on CT in a single institutional retrospective study: a propensity score matching analysis. Radiat Oncol 2018;13:200.

11. Yang JW, Choi YL. Genomic profiling of esophageal squamous cell carcinoma (ESCC)-Basis for precision medicine. Pathol Res Pract 2017;213:836-41.

12. Wang L, Yang HY, Zheng YQ. Personalized medicine of esophageal cancer. J Cancer Res Ther 2012;8:343-7.

13. Zhang L, Ma J, Han Y, et al. Targeted therapy in esophageal cancer. Expert Rev Gastroenterol Hepatol 2016;10:595-604.

14. Zhang H, Li H, Ma Q, et al. Predicting malignant transformation of esophageal squamous cell lesions by combined biomarkers in an endoscopic screening program. World J Gastroenterol 2016;22:8770-8.

15. Indinnimeo $M$, Reale MG, Cicchini C, et al. CEA, TPA, CA 19-9, SCC and CYFRA at diagnosis and in the followup of anal canal tumors. Int Surg 1997;82:275-9.

16. Mansour NM, Groth SS, Anandasabapathy S. Esophageal adenocarcinoma: screening, surveillance, and management. Annu Rev Med 2017;68:213-27.

17. Rice TW, Patil DT, Blackstone EH. 8th edition AJCC/UICC staging of cancers of the esophagus and esophagogastric junction: application to clinical practice. Ann Cardiothorac Surg 2017;6:119-30.

18. Liu J, Wang Z, Shao H, et al. Improving CT detection sensitivity for nodal metastases in oesophageal cancer with combination of smaller size and lymph node axial ratio. Eur Radiol 2018;28:188-95.
19. Giganti F, Ambrosi A, Petrone MC, et al. Prospective comparison of MR with diffusion-weighted imaging, endoscopic ultrasound, MDCT and positron emission tomography-CT in the pre-operative staging of oesophageal cancer: results from a pilot study. Br J Radiol 2016;89:20160087.

20. Lowe VJ, Booya F, Fletcher JG, et al. Comparison of positron emission tomography, computed tomography, and endoscopic ultrasound in the initial staging of patients with esophageal cancer. Mol Imaging Biol 2005;7:422-30.

21. Puli SR, Reddy JB, Bechtold ML, et al. Staging accuracy of esophageal cancer by endoscopic ultrasound: a metaanalysis and systematic review. World J Gastroenterol 2008;14:1479-90.

22. Griffin Y. Esophageal cancer: role of imaging in primary staging and response assessment post neoadjuvant therapy. Semin Ultrasound CT MR 2016;37:339-51.

23. Ba-Ssalamah A, Matzek W, Baroud S, et al. Accuracy of hydro-multidetector row CT in the local T staging of oesophageal cancer compared to postoperative histopathological results. Eur Radiol 2011;21:2326-35.

24. Kim SH, Lee JM, Han JK, et al. Three-dimensional MDCT imaging and CT esophagography for evaluation of esophageal tumors: preliminary study. Eur Radiol 2006;16:2418-26.

25. Panebianco V, Grazhdani H, Iafrate F, et al. 3D CT protocol in the assessment of the esophageal neoplastic lesions: can it improve TNM staging? Eur Radiol 2006;16:414-21.

26. Onbaş O, Eroglu A, Kantarci M, et al. Preoperative staging of esophageal carcinoma with multidetector CT and virtual endoscopy. Eur J Radiol 2006;57:90-5.

27. Pongpornsup S, Posri S, Totanarungroj K. Diagnostic accuracy of multidetector computed tomography (MDCT) in evaluation for mediastinal invasion of esophageal cancer. J Med Assoc Thai 2012;95:704-11.

28. Betancourt Cuellar SL, Sabloff B, Carter BW, et al. Early clinical esophageal adenocarcinoma (cT1): Utility of CT in regional nodal metastasis detection and can the clinical accuracy be improved? Eur J Radiol 2017;88:56-60.

29. Matthews R, Choi M. Clinical utility of positron emission tomography magnetic resonance imaging (PET-MRI) in gastrointestinal cancers. Diagnostics (Basel) 2016. doi: 10.3390/diagnostics6030035.

30. Borakati A, Razack A, Cawthorne C, et al. A comparative study of quantitative assessment with fluorine-18fluorodeoxyglucose positron-emission tomography and endoscopic ultrasound in oesophageal cancer. Nucl Med 
Commun 2018;39:628-35.

31. Hu J, Zhu D, Yang Y. Diagnostic value of $18 \mathrm{~F}$-fluorodeoxyglucose positron-emission tomography/ computed tomography for preoperative lymph node metastasis of esophageal cancer: A meta-analysis. Medicine (Baltimore) 2018;97:e13722.

32. Shi W, Wang W, Wang J, et al. Meta-analysis of 18 FDG PET-CT for nodal staging in patients with esophageal cancer. Surg Oncol 2013;22:112-6.

33. Bunting D, Bracey T, Fox B, et al. Loco-regional staging accuracy in oesophageal cancer-How good are we in the modern era? Eur J Radiol 2017;97:71-5.

34. Chatterton BE, Ho Shon I, Baldey A, et al. Positron emission tomography changes management and prognostic stratification in patients with oesophageal cancer: results of a multicentre prospective study. Eur J Nucl Med Mol Imaging 2009;36:354-61.

35. van Rossum PS, van Lier AL, Lips IM, et al. Imaging of oesophageal cancer with FDG-PET/CT and MRI. Clin Radiol 2015;70:81-95.

36. Jiang Y, Chen YL, Chen TW, et al. Is there association of gross tumor volume of adenocarcinoma of oesophagogastric junction measured on magnetic resonance imaging with $\mathrm{N}$ stage? Eur J Radiol 2019;110:181-6.

37. Goense L, Heethuis SE, van Rossum PSN, et al. Correlation between functional imaging markers derived from diffusion-weighted MRI and 18F-FDG PET/CT in esophageal cancer. Nucl Med Commun 2018;39:60-7.

38. van Rossum PS, van Hillegersberg R, Lever FM, et al. Imaging strategies in the management of oesophageal cancer: what's the role of MRI? Eur Radiol 2013;23:1753-65.

39. Zhu Y, Fu L, Jing W, et al. The value of magnetic resonance imaging in esophageal carcinoma: Tool or toy? Asia Pac J Clin Oncol 2019;15:101-7.

40. Alper F, Turkyilmaz A, Kurtcan S, et al. Effectiveness of the STIR turbo spin-echo sequence MR imaging in evaluation of lymphadenopathy in esophageal cancer. Eur J Radiol 2011;80:625-8.

41. Malik V, Harmon M, Johnston C, et al. whole body MRI in the staging of esophageal cancer--a prospective comparison with whole body 18F-FDG PET-CT. Dig Surg 2015;32:397-408.

42. Lee G, I H, Kim SJ, et al. Clinical implication of PET/ MR imaging in preoperative esophageal cancer staging: comparison with PET/CT, endoscopic ultrasonography, and CT. J Nucl Med 2014;55:1242-7.
43. Qiu B, Wang D, Yang H, et al. Combined modalities of magnetic resonance imaging, endoscopy and computed tomography in the evaluation of tumor responses to definitive chemoradiotherapy in esophageal squamous cell carcinoma. Radiother Oncol 2016;121:239-45.

44. Dewan A, Sharma SK, Dewan AK, et al. impact on radiological and pathological response with neoadjuvant chemoradiation and its effect on survival in squamous cell carcinoma of thoracic esophagus. J Gastrointest Cancer 2017;48:42-9.

45. Foley K, Findlay J, Goh V. Novel imaging techniques in staging oesophageal cancer. Best Pract Res Clin Gastroenterol 2018;36-37:17-25.

46. Ajani JA, D'Amico TA, Almhanna K, et al. Esophageal and esophagogastric junction cancers, version 1.2015. J Natl Compr Canc Netw 2015;13:194-227.

47. Lordick F, Mariette C, Haustermans K, et al. Oesophageal cancer: ESMO Clinical Practice Guidelines for diagnosis, treatment and follow-up. Ann Oncol 2016;27:v50-v57.

48. Wang L, Han C, Zhu S, et al. Investigation of using diffusion-weighted magnetic resonance imaging to evaluate the therapeutic effect of esophageal carcinoma treatment. Oncol Res Treat 2014;37:112-6.

49. Alfieri R, Pintacuda G, Cagol M, et al. Oesophageal cancer: assessment of tumour response to chemoradiotherapy with tridimensional CT. Radiol Med 2015;120:430-9.

50. Wakatsuki K, Matsumoto S, Migita K, et al. Usefulness of computed tomography density of a tumor in predicting the response of advanced esophageal cancer to preoperative chemotherapy. Surgery 2017;162:823-35.

51. Djuric-Stefanovic A, Micev M, Stojanovic-Rundic S, et al. Absolute CT perfusion parameter values after the neoadjuvant chemoradiotherapy of the squamous cell esophageal carcinoma correlate with the histopathologic tumor regression grade. Eur J Radiol 2015;84:2477-84.

52. Ge X, Yu J, Wang Z, et al. Comparative study of dual energy CT iodine imaging and standardized concentrations before and after chemoradiotherapy for esophageal cancer. BMC Cancer 2018;18:1120.

53. Cremonesi M, Garibaldi C, Timmerman R, et al. Interim (18)F-FDG-PET/CT during chemo-radiotherapy in the management of oesophageal cancer patients. A systematic review. Radiother Oncol 2017;125:200-12.

54. Zschaeck S, Hofheinz F, Zophel K, et al. Increased FDG uptake on late-treatment PET in non-tumour-affected oesophagus is prognostic for pathological complete response and disease recurrence in patients undergoing neoadjuvant radiochemotherapy. Eur J Nucl Med Mol 
Imaging 2017;44:1813-22.

55. Findlay JM, Bradley KM, Wang LM, et al. Predicting pathologic response of esophageal cancer to neoadjuvant chemotherapy: the implications of metabolic nodal response for personalized therapy. J Nucl Med 2017;58:266-75.

56. Kroese TE, Goense L, van Hillegersberg R, et al. Detection of distant interval metastases after neoadjuvant therapy for esophageal cancer with 18F-FDG PET(/CT): a systematic review and meta-analysis. Dis Esophagus 2018. doi: 10.1093/dote/doy055.

57. De Cobelli F, Giganti F, Orsenigo E, et al. Apparent diffusion coefficient modifications in assessing gastrooesophageal cancer response to neoadjuvant treatment: comparison with tumour regression grade at histology. Eur Radiol 2013;23:2165-74.

58. Aoyagi T, Shuto K, Okazumi S, et al. Apparent diffusion coefficient values measured by diffusion-weighted imaging predict chemoradiotherapeutic effect for advanced esophageal cancer. Dig Surg 2011;28:252-7.

59. van Rossum PS, van Lier AL, van Vulpen M, et al. Diffusion-weighted magnetic resonance imaging for the prediction of pathologic response to neoadjuvant chemoradiotherapy in esophageal cancer. Radiother Oncol 2015;115:163-70.

60. Sun NN, Liu C, Ge XL, et al. Dynamic contrast-enhanced MRI for advanced esophageal cancer response assessment after concurrent chemoradiotherapy. Diagn Interv Radiol 2018;24:195-202.

61. Heethuis SE, Goense L, van Rossum PSN, et al. DW-MRI and DCE-MRI are of complementary value in predicting pathologic response to neoadjuvant chemoradiotherapy for esophageal cancer. Acta Oncol 2018;57:1201-8.

62. Heiden BT, Patel N, Nancarrow DJ, et al. Positron emission tomography $18 \mathrm{~F}$-fluorodeoxyglucose uptake correlates with KRAS and EMT gene signatures in operable esophageal adenocarcinoma. J Surg Res 2018;232:621-8.

63. Tan X, Ma Z, Yan L, et al. Radiomics nomogram outperforms size criteria in discriminating lymph node metastasis in resectable esophageal squamous cell carcinoma. Eur Radiol 2019;29:392-400.

64. Klaassen R, Larue R, Mearadji B, et al. Feasibility of CT radiomics to predict treatment response of individual liver metastases in esophagogastric cancer patients. PLoS One 2018;13:e0207362.

65. Hou Z, Ren W, Li S, et al. Radiomic analysis in contrast-enhanced CT: predict treatment response to chemoradiotherapy in esophageal carcinoma. Oncotarget 2017;8:104444-54.

66. Ganeshan B, Skogen K, Pressney I, et al. Tumour heterogeneity in oesophageal cancer assessed by CT texture analysis: preliminary evidence of an association with tumour metabolism, stage, and survival. Clin Radiol 2012;67:157.

67. Yip C, Landau D, Kozarski R, et al. Primary esophageal cancer: heterogeneity as potential prognostic biomarker in patients treated with definitive chemotherapy and radiation therapy. Radiology 2014;270:141-8.

68. Sah BR, Owczarczyk K, Siddique M, et al. Radiomics in esophageal and gastric cancer. Abdom Radiol (NY) 2019;44:2048-58.

69. Larue RTHM, Klaassen R, Jochems A, et al. Pretreatment CT radiomics to predict 3-year overall survival following chemoradiotherapy of esophageal cancer. Acta Oncol 2018;57:1475-81.

70. Tixier F, Le Rest CC, Hatt M, et al. Intratumor heterogeneity characterized by textural features on baseline 18F-FDG PET images predicts response to concomitant radiochemotherapy in esophageal cancer. J Nucl Med 2011;52:369-78.

71. Beukinga RJ, Hulshoff JB, van Dijk LV, et al. Predicting Response to Neoadjuvant Chemoradiotherapy in Esophageal Cancer with Textural Features Derived from Pretreatment (18)F-FDG PET/CT Imaging. J Nucl Med 2017;58:723-9.

72. Nakajo M, Jinguji M, Nakabeppu $Y$, et al. Texture analysis of (18)F-FDG PET/CT to predict tumour response and prognosis of patients with esophageal cancer treated by chemoradiotherapy. Eur J Nucl Med Mol Imaging 2017;44:206-14.

73. Paul D, Su R, Romain M, et al. Feature selection for outcome prediction in oesophageal cancer using genetic algorithm and random forest classifier. Comput Med Imaging Graph 2017;60:42-9.

74. Foley KG, Hills RK, Berthon B, et al. Development and validation of a prognostic model incorporating texture analysis derived from standardised segmentation of PET in patients with oesophageal cancer. Eur Radiol 2018;28:428-36.

75. van Rossum PS, Fried DV, Zhang L, et al. The incremental value of subjective and quantitative assessment of 18F-FDG PET for the prediction of pathologic complete response to preoperative chemoradiotherapy in esophageal cancer. J Nucl Med 2016;57:691-700.

76. Yip SS, Coroller TP, Sanford NN, et al. Use of registration-based contour propagation in texture analysis 
for esophageal cancer pathologic response prediction. Phys Med Biol 2016;61:906-22.

77. Beukinga RJ, Hulshoff JB, Mul VEM, et al. Prediction of Response to Neoadjuvant Chemotherapy and Radiation Therapy with Baseline and Restaging (18)F-FDG PET Imaging Biomarkers in Patients with Esophageal Cancer. Radiology 2018;287:983-92.

78. Qu J, Shen C, Qin J, et al. The MR radiomic signature can predict preoperative lymph node metastasis in patients with esophageal cancer. Eur Radiol 2019;29:906-14.

79. Mariette C, Balon JM, Piessen G, et al. Pattern of recurrence following complete resection of esophageal carcinoma and factors predictive of recurrent disease. Cancer 2003;97:1616-23.

80. Blom RL, Lagarde SM, van Oudenaarde K, et al. Survival after recurrent esophageal carcinoma has not improved over the past 18 years. Ann Surg Oncol 2013;20:2693-8.

81. Goense L, van Rossum PS, Reitsma JB, et al. Diagnostic performance of (1)(8)F-FDG PET and PET/CT for the detection of recurrent esophageal cancer after treatment with curative intent: a systematic review and meta-analysis. J Nucl Med 2015;56:995-1002.

82. Kato H, Miyazaki T, Nakajima M, et al. Value of positron emission tomography in the diagnosis of recurrent oesophageal carcinoma. Br J Surg 2004;91:1004-9.

83. Teyton P, Metges JP, Atmani A, et al. Use of positron emission tomography in surgery follow-up of esophageal cancer. J Gastrointest Surg 2009;13:451-8.

84. Kudou M, Shiozaki A, Fujiwara H, et al. Efficacy of PET-CT in the diagnosis and treatment of recurrence

Cite this article as: Elsherif SB, Andreou S, Virarkar M, Soule E, Gopireddy DR, Bhosale PR, Lall C. Role of precision imaging in esophageal cancer. J Thorac Dis 2020;12(9):5159-5176. doi: $10.21037 /$ jtd.2019.08.15 after esophageal cancer surgery. Anticancer Res 2016;36:5473-80.

85. Yamada $\mathrm{H}$, Hosokawa $M$, Itoh $\mathrm{K}$, et al. Diagnostic value of (1)(8)F-FDG PET/CT for lymph node metastasis of esophageal squamous cell carcinoma. Surg Today 2014;44(7):1258-65.

86. Kantarci M, Polat P, Alper F, et al. Comparison of CT and MRI for the diagnosis recurrent esophageal carcinoma after operation. Dis Esophagus 2004;17(1):32-7.

87. Shuto K, Saito H, Ohira G, et al. [Diffusion-weighted MR imaging for postoperative nodal recurrence of esophageal squamous cell cancer in comparison with FDG-PET]. Gan To Kagaku Ryoho 2009;36:2468-70.

88. Okumura H, Natsugoe S, Matsumoto M, et al. The predictive value of $\mathrm{p} 53, \mathrm{p} 53 \mathrm{R} 2$, and $\mathrm{p} 21$ for the effect of chemoradiation therapy on oesophageal squamous cell carcinoma. Br J Cancer 2005;92:284-9.

89. Li Y, Zschaeck S, Lin Q, et al. Metabolic parameters of sequential 18F-FDG PET/CT predict overall survival of esophageal cancer patients treated with (chemo-) radiation. Radiat Oncol 2019;14:35.

90. Chang S, Kim SJ. Prediction of recurrence and mortality of locally advanced esophageal cancer patients using pretreatment F-18 FDG PET/CT parameters: intratumoral heterogeneity, SUV, and volumetric parameters. Cancer Biother Radiopharm 2016;31:1-6.

91. Sugawara K, Yamashita H, Uemura Y, et al. Preoperative lymph node status on computed tomography influences the survival of pT1b, T2 and T3 esophageal squamous cell carcinoma. Surg Today 2019;49:378-86. 